\title{
The neurofunctional consequences of amphetamine sensitisation on human working memory function - an $\mathrm{PMRI}$ investigation Owen O'Daly*, Panayiota Michalopoulou, Gabrielle Samson, Cori Smee, Derek Tracy, Robin Murray and Sukhwinder Shergill
}

Address: The Institute of Psychiatry, London, UK

* Corresponding author

from International Society on Brain and Behaviour: 2nd International Congress on Brain and Behaviour Thessaloniki, Greece. 17-20 November 2005

Published: 28 February 2006

Annals of General Psychiatry 2006, 5(SuppI I):S88 doi:I0.I I86/I744-859X-5-SI-S88

\section{Background}

Patients with schizophrenia and chronic substance abusers often display deficits in working memory function linked to prefrontal cortical dysfunction. Cortical dopamine transmission strongly influences the efficiency of capacity-constrained prefrontal activity. A recent fMRI study showed that amphetamine modulates cortical processing in line with an inverted-U type dose-response function. Repeated intermittent exposure to amphetamine in primates has been shown to result in augmentation of the behavioural response elicited by the drug (eg: hyperlocomotion) known as sensitisation. Chronic treatment produces cognitive deficits similar to those observed in clinical populations and sensitisation is a well established animal model of behavioural and neurochemical aspects of schizophrenia. In this study we use fMRI to examine the behavioural consequences and neural correlates of working memory function in healthy volunteers displaying behavioural sensitisation.

\section{Materials and methods}

Twenty-two participants were recruited and randomly assigned to one of two groups. Group 1 received three doses of d-Amphetamine with an inter-dose interval of 48 hours, and then a final fourth dose of d-Amphetamine after a 14-day withdrawal period. Group 2 followed the same experimental design but received only placebo in a double blind design. All participants underwent fMRI scanning on two occasions (session 1 and session 4) three apart while performing a parametric verbal working memory task. Analysis was carried out using neuroimgaing statisitical SPM2.

\section{Results}

Amphetamine sensitisation results in an alteration in the cortical response to amphetamine in the DLPFC in accord with previous animal work. In individuals displaying sensitisation cortical response to amphetamine was dependent upon the initial response and was characterised by a shift to the left of the inverted-U dose-response curve, resulting in a reduction in DLPFC activation.

\section{Discussion}

The demonstration of neurofunctional changes in sensitised humans which accord with observations in animals validates these models of working memory dysfunction. The cortical response to amphetamine following sensitisation also mirrors the hypofrontality observed in clinical populations lending further support to the psychostimulant sensitisation model. These results also hint at the potential use of low-dose psychostimulant exposure as a treatment for the cognitive deficits observed in a number of psychiatric illnesses and the cognitive decline seen with aging. 